\title{
Multimode Storage and Retrieval of Microwave Fields in a Spin Ensemble
}

\author{
C. Grezes, ${ }^{1}$ B. Julsgaard, ${ }^{2}$ Y. Kubo, ${ }^{1,7}$ M. Stern, ${ }^{1}$ T. Umeda, ${ }^{3}$ J. Isoya, ${ }^{4}$ H. Sumiya, ${ }^{5}$ H. Abe, ${ }^{6}$ S. Onoda, ${ }^{6}$ \\ T. Ohshima, ${ }^{6}$ V. Jacques, ${ }^{7,8}$ J. Esteve, ${ }^{9}$ D. Vion, ${ }^{1}$ D. Esteve, ${ }^{1}$ K. Mølmer, ${ }^{2}$ and P. Bertet ${ }^{1}$ \\ ${ }^{1}$ Quantronics group, SPEC (CNRS URA 2464), IRAMIS, DSM, CEA-Saclay, 91191 Gif-sur-Yvette, France \\ ${ }^{2}$ Department of Physics and Astronomy, Aarhus University, \\ Ny Munkegade 120, DK-8000 Aarhus C, Denmark \\ ${ }^{3}$ Institute of Applied Physics, University of Tsukuba, Tsukuba 305-8573, Japan \\ ${ }^{4}$ Research Center for Knowledge Communities, University of Tsukuba, Tsukuba 305-8550, Japan \\ ${ }^{5}$ Sumitomo Electric Industries Ltd., Itami 664-001, Japan \\ ${ }^{6}$ Japan Atomic Energy Agency, Takasaki 370-1292, Japan \\ ${ }^{7}$ Laboratoire de Photonique Quantique et Moléculaire, ENS Cachan and CNRS UMR 8537, \\ 94235 Cachan, France \\ ${ }^{8}$ Laboratoire Aimé Cotton, CNRS, Université Paris-Sud and ENS Cachan, 91405 Orsay, France \\ ${ }^{9}$ Laboratoire Kastler Brossel, ENS, UPMC-Paris 6, CNRS, 24 rue Lhomond, 75005 Paris, France \\ (Received 28 January 2014; revised manuscript received 14 April 2014; published 16 June 2014)
}

\begin{abstract}
A quantum memory at microwave frequencies, able to store the state of multiple superconducting qubits for long times, is a key element for quantum information processing. Electronic and nuclear spins are natural candidates for the storage medium as their coherence time can be well above $1 \mathrm{~s}$. Benefiting from these long coherence times requires one to apply the refocusing techniques used in magnetic resonance, a major challenge in the context of hybrid quantum circuits. Here, we report the first implementation of such a scheme, using ensembles of nitrogen-vacancy centers in diamond coupled to a superconducting resonator, in a setup compatible with superconducting qubit technology. We implement the active reset of the nitrogen-vacancy spins into their ground state by optical pumping and their refocusing by Hahn-echo sequences. This enables the storage of multiple microwave pulses at the picowatt level and their retrieval after up to $35 \mu \mathrm{s}$, a 3 orders of magnitude improvement compared to previous experiments.
\end{abstract}

DOI: 10.1103/PhysRevX.4.021049

\section{INTRODUCTION}

The ability to store a quantum state over long times is a desirable feature in many quantum information protocols. In the optical domain, quantum memories are necessary to implement the quantum repeaters needed for future longdistance quantum networks and are the object of active research [1-5]. Quantum memories at microwave frequencies have also become of great interest in recent years because of the development of superconducting qubits, which have their resonance frequency in the $\mathrm{GHz}$ range, in the perspective of implementing holographic quantum computing [6-8]. For such schemes, the memory should act as an ideal multiqubit register, able to store the state of large numbers of qubits over long times and to retrieve them on demand.

Spin ensembles have emerged as promising candidates for such a microwave quantum memory because of their

Published by the American Physical Society under the terms of the Creative Commons Attribution 3.0 License. Further distribution of this work must maintain attribution to the author(s) and the published article's title, journal citation, and DOI.
Subject Areas: Condensed Matter Physics,

Quantum Information long coherence time [9-12] and because a spin ensemble withstands many orthogonal collective modes that are well suited to store multiple qubit states. Existing proposals $[13,14]$ (inspired by optical quantum memory protocols $[4,5])$ proceed in two distinct steps. In the write step, the microwave field prepared in a well-defined quantum state $|\psi\rangle$ (for instance, by a superconducting qubit) is absorbed by the spin ensemble. This generates a transverse magnetization that decays rapidly in a time $T_{2}^{*}$ due to the spread of resonance frequencies in the ensemble. Given the weakness of the coupling constant of a single spin to the microwave field, efficient absorption requires embedding the ensemble in a high-quality factor microwave resonator in order to reach the so-called high-cooperativity regime [15-19]. The second step (read) of the memory operation consists of retrieving the initial state. It relies on the principle of Hahn echoes [20]: dephasing due to inhomogeneous broadening can be counteracted by applying a $\pi$ pulse to the spins at time $\tau$, which acts as a time reversal and, thus, causes all the magnetic dipoles to return in phase (refocus) at time $2 \tau$. Quantum memory proposals $[13,14]$ combine two consecutive refocusing $\pi$ pulses applied to the spins with dynamical tuning of the resonator frequency and 
quality factor, thereby retrieving the amplitude of the input microwave field while preventing the addition of noise, as required for a high-fidelity memory performance [21]. The maximum storage time of the memory is approximately the Hahn-echo decay time $T_{2}$, so that the maximal number of stored quantum states is of order $T_{2} / T_{2}^{*}$, a figure which can be very large in many spin systems.

The write step of this protocol (quantum state transfer) has been demonstrated at the single-photon level in recent experiments [22,23]; the remaining obstacle to a multimode microwave quantum memory is therefore the implementation of Hahn-echo refocusing sequences at the quantum level in a hybrid quantum circuit. The object of this work is precisely to identify the challenges posed by this task and to demonstrate experimentally that they can be solved. For simplicity, we consider a protocol simpler than the full quantum memory [14] but which constitutes an essential building block: the two-pulse echo (2PE). As depicted in Fig. 1(a), the 2PE consists in storing weak pulses $\theta_{i}$ into the spin ensemble at times $t_{i}$ and applying a single refocusing pulse at time $\tau$, which triggers the emission of echo pulses $e_{i}$ at times $2 \tau-t_{i}$ (therefore, in reverse order) in the detection waveguide [24].

Performing the $2 \mathrm{PE}$ at the quantum level imposes a number of requirements that represent experimental challenges. For quantum states to be well defined, thermal excitations should be absent from the system. This implies both that the spin ensemble has a high degree of polarization and that the microwave field is in its ground state with high probability, which can only be achieved if the experiments are performed at millikelvin temperatures. At these temperatures, however, spins tend to relax very slowly towards their ground state, and an active spin reset is therefore needed in order to repeat the experimental (a)

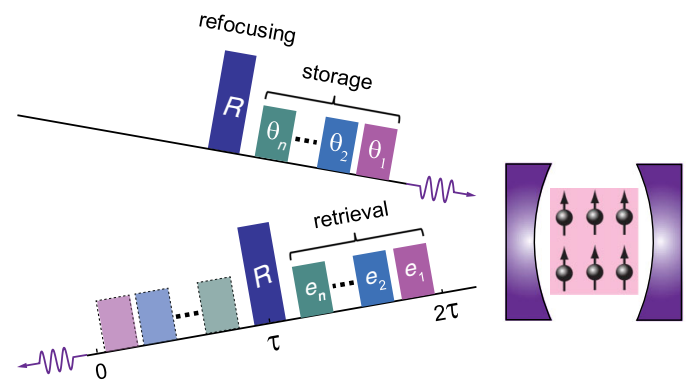

(b)

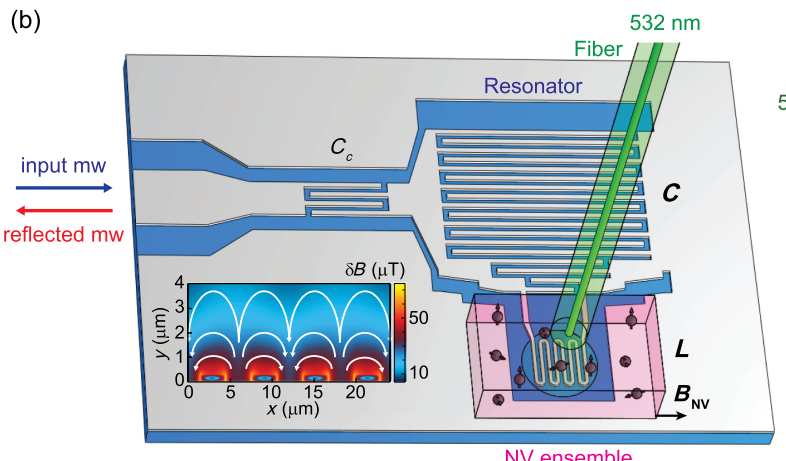

(c)

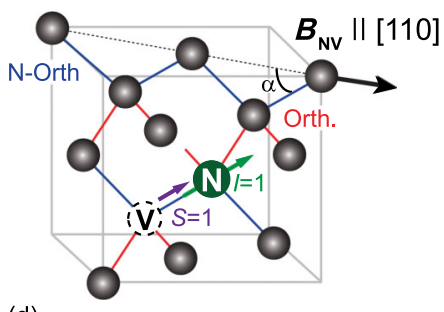

(d)

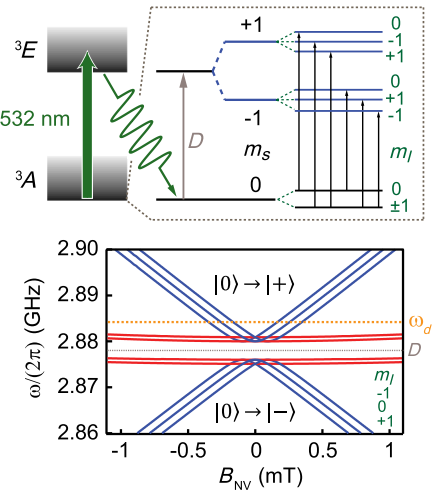

FIG. 1. Principle of the experiment. (a) Scheme of the multimode two-pulse echo (2PE) protocol applied to an ensemble of spins placed in an electromagnetic cavity: successive low-power microwave pulses $\theta_{i}$ are stored in the spin ensemble. A refocusing pulse $R$ acts as time reversal for the spins and triggers the retrieval of the stored pulses as echoes $e_{i}$ in reverse order. Top and bottom time lines show the applied and the reflected and echo signals, respectively. (b) Setup placed in a dilution refrigerator: the cavity is a lumpedelement parallel $L C$ resonator in niobium coupled to a coplanar waveguide by a capacitor $C_{c}$. It consists of an interdigitated capacitor $C$ and a meander wire inductor $L$ creating the ac magnetic field shown in the inset, for a $10 \mu \mathrm{W}$ incident microwave power at resonance. The spin ensemble consists of NV centers in a diamond monocrystal pressed on top of the inductor. Laser pulses can be shone on it through an optical fiber glued to its top face. A tunable dc magnetic field $B_{\mathrm{NV}}$ is applied parallel to the [110] direction of the crystal. (c) Negatively charged NV centers in diamond consist of a nitrogen atom next to a vacancy of the diamond lattice, having trapped an electron. Their electronic spin $S=1$ is coupled by hyperfine interaction to the nitrogen nuclear spin $I=1$ (for the ${ }^{14} \mathrm{~N}$ isotope). Half of the electronic spins (subensemble denoted N-Orth. in blue) make an angle $\alpha=35.3^{\circ}$ with $B_{\mathrm{NV}}$, whereas the other half (subensemble Orth. in red) is orthogonal to the field. (d) NV simplified energy diagram (top) showing the ground ${ }^{3} A$ and the excited ${ }^{3} E$ electronic states as well as the Zeeman and hyperfine structure of ${ }^{3} A$, with $D / 2 \pi=2.8775 \mathrm{GHz}$ the zero-field splitting. (bottom) Magnetic field dependence of the allowed transitions for both N-Orth. (blue) and Orth. (red) subensembles, showing, respectively, a linear and a quadratic Zeeman effect. NVs can be optically repumped in their $m_{S}=0$ ground state by application of green (532 nm) laser pulses exciting the ${ }^{3} A-{ }^{3} E$ transition. 
sequence at a reasonable rate $(>1 \mathrm{~Hz})$ as required by experiments at the single-photon level. Then, applying refocusing pulses to the spins requires large microwave powers potentially incompatible with the detection of quantum fields. Finally, the echo emitted by the spins should faithfully restore the initial field, which implies that the echo recovery efficiency $E$, which we define as the ratio of the energy radiated during the echo to the energy of the incoming pulse, should be close to 1 . To summarize, reaching the quantum regime requires a mean excitation per mode (both microwave and spin) $n_{\text {mw,sp }} \ll 1$, input microwave fields with intracavity photon number $\bar{n} \approx 1$, and an echo efficiency $E$ close to 1 .

These stringent requirements have never been met in an experiment, by far. The multimode character of the 2PE has been recently benchmarked in the classical regime [25] with an ensemble of phosphorus donors in silicon at $10 \mathrm{~K}$ in the three-dimensional microwave cavity of an electron paramagnetic resonance spectrometer. That experiment reached $n_{\mathrm{mw}, \mathrm{sp}} \approx 20, \bar{n} \approx 10^{14}$, and an echo recovery efficiency $E \approx 10^{-10}$. Here, we use negatively charged NV centers in diamond, which are color centers consisting of a substitutional nitrogen atom sitting next to a vacancy of the lattice [see Fig. 1(c)] with properties suitable for a quantum memory. Indeed, their spin triplet $(S=1)$ electronic ground state has a long coherence time [9] and can be optically repumped in the spin ground state $\left|m_{S}=0\right\rangle$ [see Figs. 1(c) and 1(d)] as a result of spin-dependent intersystem crossing from the electronic excited triplet state to a metastable singlet state [not shown in Fig. 1(d)] [26]. We revisit the 2PE protocol with an ensemble of NV centers at $400 \mathrm{mK}$ coupled to a planar superconducting resonator, in a setup compatible with hybrid quantum circuits, with active reset of the spin at the beginning of each experimental sequence, and we demonstrate the storage of multiple pulses at the picowatt level into orthogonal collective modes of the spin ensemble for $35 \mu \mathrm{s}, 3$ orders of magnitude longer than in earlier experiments [27]. Our experiment reaches $n_{\mathrm{mw}} \approx 3, n_{\mathrm{sp}} \approx 0.1, \bar{n} \approx 100$, and $E \approx 2 \times 10^{-4}$, and, therefore, comes closer to the quantum regime than previous work by several orders of magnitude. We quantitatively identify the present limitations and show that they can be solved in future experiments, opening the way to the implementation of quantum memory protocols.

\section{EXPERIMENTAL SETUP AND NV HAMILTONIAN}

The experimental setup is sketched in Fig. 1(b) (see also the Supplemental Material [28]). A diamond crystal homogeneously doped with $\mathrm{NV}$ centers $\left(\left[\mathrm{NV}^{-}\right] \approx 2 \mathrm{ppm}\right)$ is glued on top of the inductance of a planar superconducting $L C$ resonator cooled in a dilution refrigerator. For optical pumping, $532 \mathrm{~nm}$ laser light is injected through a singlemode optical fiber, glued on top of the crystal, $1.5 \mathrm{~mm}$ above the resonator inductance. A magnetic field $\vec{B}_{\mathrm{NV}}$ is applied parallel to the chip along the [110] crystalline axis [see Fig. 1(c)].

NV centers in their ground state are described [29] by the Hamiltonian $H_{\mathrm{NV}} / \hbar=D S_{z}^{2}+E\left(S_{x}^{2}-S_{y}^{2}\right)+A_{z} S_{z} I_{z}+$ $\gamma_{e} \vec{B}_{\mathrm{NV}} \cdot \vec{S}+Q\left[I_{z}^{2}-I(I+1) / 3\right]$, with $\vec{S}(\vec{I})$ the spin operator of the $S=1 \mathrm{NV}$ electronic spin (the $I=1$ nitrogen nuclear spin), $D / 2 \pi=2.8775 \mathrm{GHz}$ the zero-field splitting between states $m_{S}=0$ and $m_{S}= \pm 1, A_{z}=-2.1 \mathrm{MHz}$ the hyperfine coupling, and $Q=-5 \mathrm{MHz}$ the nuclear quadrupole momentum [30]. Local electric field and strain couple the spin eigenstates $\left|m_{S}= \pm 1\right\rangle$ with strength $E$ [31]. The energy eigenstates $| \pm\rangle$, shown in Fig. 1(d), are thus linear combinations of states $\left|m_{S}= \pm 1\right\rangle$; in particular, at zero magnetic field, states $| \pm\rangle=\left(\left|m_{S}=+1\right\rangle \pm\left|m_{S}=-1\right\rangle\right) / \sqrt{2}$ are separated in energy by $2 E$. In the experiment, we use transitions between the spin ground state $\left|m_{S}=0\right\rangle$ and the two excited states $| \pm\rangle$ at frequencies close to the zero-field splitting.

The resonator is capacitively coupled to measurement lines through which microwave signals are applied, with the amplitude and phase of the reflected field detected by homodyne demodulation after amplification at $4 \mathrm{~K}$. The reflection coefficient $S_{11}$, shown in Figs. 2(a) and 2(b), yields the resonator frequency $\omega_{c} / 2 \pi=2.88 \mathrm{GHz}$ and quality factor $Q=80$. Such a low $Q$ was chosen to avoid spin relaxation by superradiant spontaneous emission after excitation by the refocusing pulse [32]. Dips in $\left|S_{11}\right|$ are due to absorption by the NVs, as evidenced by their dependence on $B_{\mathrm{NV}}$.

\section{ACTIVE RESET OF THE SPINS}

To demonstrate optical repumping of the NVs in $\left|m_{S}=0\right\rangle$, we probe the spin polarization after a laser pulse of power $P_{L}$ and duration $T_{L}$ by measuring the absorption of a microwave pulse at $\omega_{d} / 2 \pi=2.884 \mathrm{GHz}$. In addition to repumping the spins, the laser generates quasiparticles in the superconductor and carriers in the silicon substrate. We thus introduce a delay of $300 \mu$ s between the two pulses for these excitations to relax. In order to start from a reproducible spin polarization, a strong microwave pulse is applied before the laser pulse, which saturates all the spins at the beginning of each sequence [see Fig. 2(c)].

The results are shown in Fig. 2(d) for $P_{L}=1.5 \mathrm{~mW}$. Without laser pulse, the reflected pulse amplitude is independent of $B_{\mathrm{NV}}$, proving that the spins are efficiently saturated by the initial microwave pulse. For nonzero $T_{L}$, absorption peaks with the triplet shape characteristic of the NV hyperfine structure are observed, indicating sizable NV polarization. To quantify the effect, we convert the absorption signal into the imaginary part of the spin susceptibility $\chi^{\prime \prime}\left(T_{L}, B_{\mathrm{NV}}\right)$ [see Fig. 2(e) and the Supplemental Material [28]], which yields the relative spin polarization $p\left(T_{L}\right)=\chi^{\prime \prime}\left(T_{L}, B_{\mathrm{NV}}\right) / \chi^{\prime \prime}\left(T_{\max }, B_{\mathrm{NV}}\right)$, with $T_{\max }$ the maximum repumping time. The polarization increases with $T_{L}$ and then saturates [see Figs. 3(a) and 3b)], which shows 

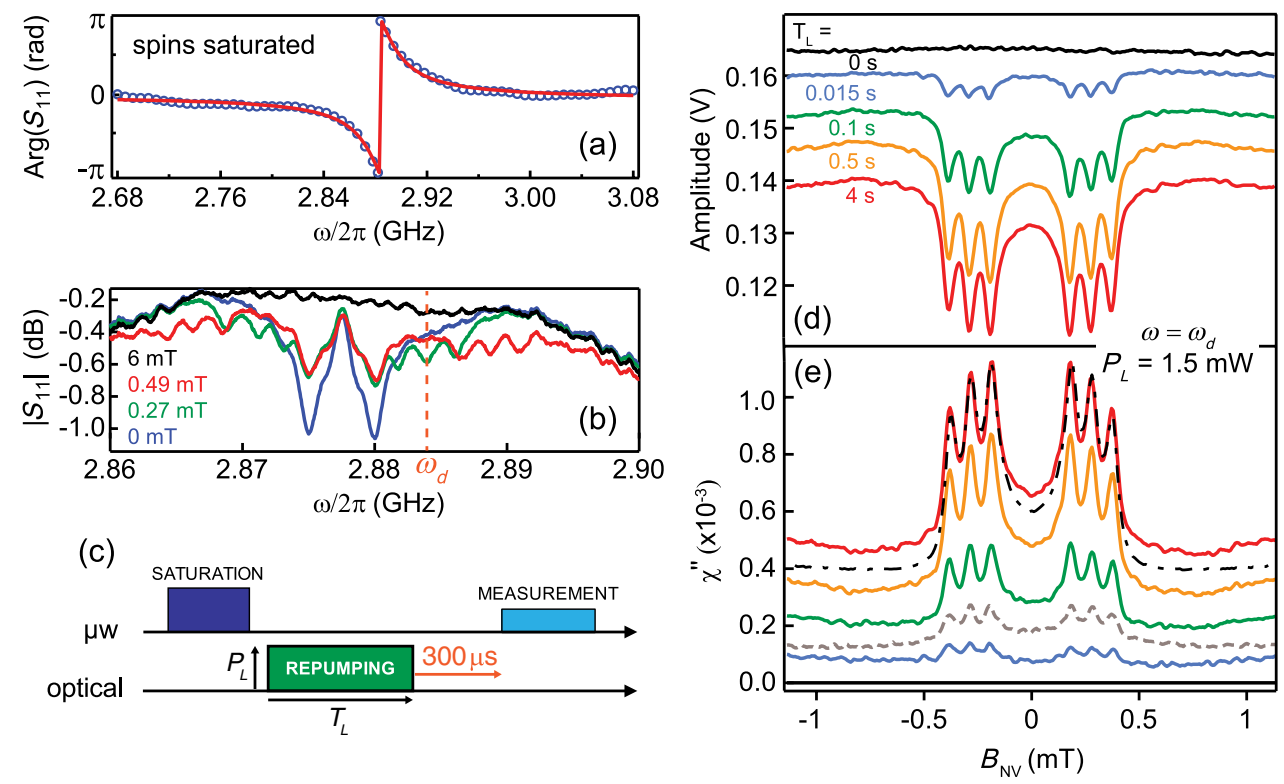

FIG. 2. Spectroscopic signals and optical repumping. (a) Measured (open circles) and fitted (solid line) phase of the reflection coefficient $S_{11}$ showing the resonator resonance at $\omega_{0} / 2 \pi=2.88 \mathrm{GHz}$ with quality factor $Q=80$, when the spins are saturated and do not contribute to the signal. (b) Measured reflection coefficient modulus $\left|S_{11}\right|$ around the center of the resonator line, showing the absorption by the spins for different magnetic fields. Top line ( $6 \mathrm{mT}$, black) corresponds to all spins (Orth. and N-Orth.) being far detuned and shows no absorption. Other lines show several absorption peaks moving with magnetic field (subensemble N-Orth.) or not (subensemble Orth). (c) Optical reset of the NV center spins. The spins are first saturated by a 20- $\mu$ s-long microwave pulse with frequency $\omega_{d}$ and applied power $-24 \mathrm{dBm}$; they are then optically repumped to their ground state with a laser pulse of power $P_{L}$ and duration $T_{L}$. After letting the system cool down during $300 \mu \mathrm{s}$, the reflected amplitude of an applied weak (-132 dBm) 20-ms-long measurement pulse at $\omega_{d} / 2 \pi=2.884 \mathrm{GHz}$ is measured. (d) Reflected amplitude for $P_{L}=1.5 \mathrm{~mW}$ and different $T_{L}$. The curves show the hyperfine split $m_{S}=0$ to $m_{S}= \pm 1$ spectroscopic transitions of the N-Orth. subensemble, with an amplitude that increases with $T_{L}$ because of increasing spin repolarization. (e) Corresponding imaginary part $\chi^{\prime \prime}\left(B_{\mathrm{NV}}\right)$ of the spin susceptibility. In addition, the dashed and dashdotted lines show, respectively, $\chi^{\prime \prime}\left(B_{\mathrm{NV}}\right)$ measured at thermal equilibrium (30 mK, no saturating nor optical pulse) and calculated (see the Supplemental Material for methods and Figs. S3 and S4 [28]) and rescaled by a global factor to match the experiment at $T_{L}=4 \mathrm{~s}$.
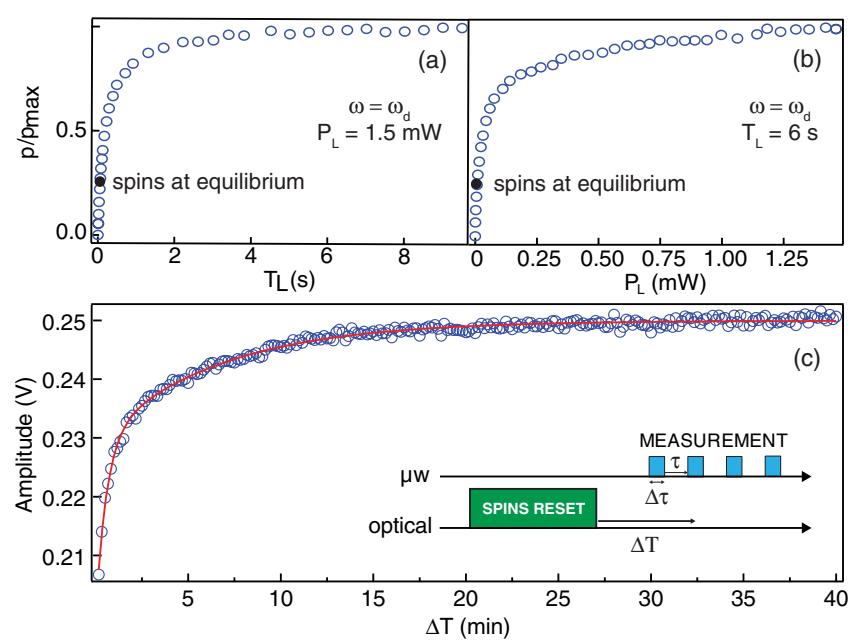

FIG. 3. Spin reset efficiency and relaxation. (a),(b) Relative spin polarization dependence on $T_{L}$ for $P_{L}=1.5 \mathrm{~mW}$ and on $P_{L}$ for $T_{L}=6 \mathrm{~s}$. The experimental sequence is shown in Fig. 2(c). (c) $\mathrm{NV}$ spin relaxation time measurement. A series of $\Delta \tau=20 \mathrm{~ms}$ weak microwave pulses $(-120 \mathrm{dBm})$ at $\omega_{d} / 2 \pi=2.884 \mathrm{GHz}$, separated by $\tau=10 \mathrm{~s}$, is applied following optical reset of the spins. Blue dots are the average reflected amplitude of each pulse. A biexponential fit (red solid line) yields $T_{1, a}=35 \mathrm{~s}$ and $T_{1, b}=395 \mathrm{~s}$. that the spins reach the maximum polarization allowed by optical pumping at $532 \mathrm{~nm}$, close to $90 \%$ according to earlier work [33]. The refrigerator cold stage was heated to $400 \mathrm{mK}$ due to laser power; all of the following results were obtained under these conditions. Better alignment of the fiber with the resonator should reduce the power needed by 2 orders of magnitude.

Using the optical pumping, we measure the energy relaxation of the spins. The spins are first repumped, after which a series of a $20 \mathrm{~ms}$ resonant probe microwave pulse separated by $10 \mathrm{~s}$ are applied. The average reflected amplitude of each pulse is plotted in Fig. 3(c) and shows a biexponential response with time constants $T_{1, a}=35 \mathrm{~s}$ and $T_{1, b}=395 \mathrm{~s}$, similar to recent measurements [18]. These very long values confirm the need of actively resetting the spins for operating a quantum memory.

\section{PULSED RESPONSE OF THE SPINS}

As a first step towards the application of refocusing pulses to the spins, we measure their time-domain response to microwave pulses of varying power. The experiments are performed at $B_{\mathrm{NV}}=0 \mathrm{mT}$. The zero-field spin 
susceptibility $\chi^{\prime \prime}(\omega)$ [see Fig. 4(a)] shows two broad peaks corresponding to the $|0\rangle \rightarrow|-\rangle$ and $|0\rangle \rightarrow|+\rangle$ transitions. The width of these peaks is governed by the inhomogeneity of local electric fields and strain acting on the NVs, which results in a broad distribution of $E$, causing the hyperfine structure to be barely resolved, as seen in Fig. 4(a). On the $|0\rangle \rightarrow|+\rangle$ transition, the spin absorption reaches a maximum at $\omega_{e} / 2 \pi=2.8795 \mathrm{GHz}$, which we will use as the frequency of all microwave pulses in the following. Square microwave pulses of varying input power $P_{\text {in }}$ are sent to the sample, and their reflected amplitude $A$ is measured. The data are shown in Figs. 4(b) and 4(c), rescaled by $\sqrt{P_{\text {in }}}$, and compared to the reflected amplitude of the same microwave pulse with the spins initially saturated by a strong pulse. At low power (the linear regime), after an initial transient where resonator and spins exchange energy, $A$ reaches half of the saturated value in steady state, indicating that the spins absorb $\approx 75 \%$ of the incoming power. The steady-state value of $A$ increases with incoming power, indicating reduced spin absorption caused by progressive saturation of the ensemble. Note that no clear Rabi oscillations are observed. This is due to the spatial inhomogeneity of the microwave field
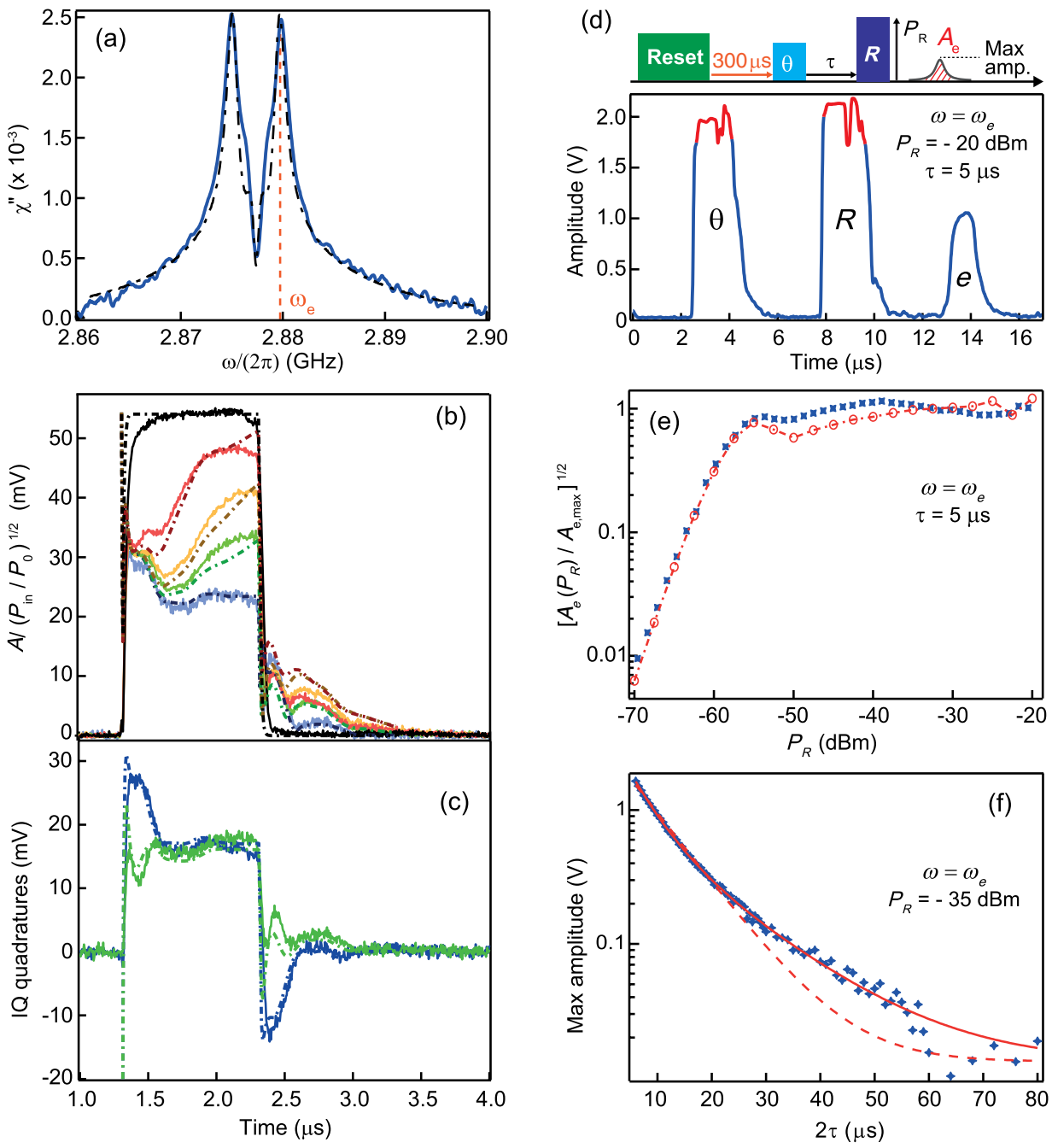

FIG. 4. (a) Measured (solid line) and computed (dash-dotted line) imaginary part $\chi^{\prime \prime}(\omega)$ of the spin susceptibility at $B_{\mathrm{NV}}=0 \mathrm{mT}$. The calculated curve (see Supplemental Material [28]) was rescaled by a global factor to match the experiment. (b) Reflected field amplitude $A$ for a square input microwave pulse of power $P_{\text {in }}$. Solid lines are experimental data with $P_{\text {in }}=-90,-60,-55$, and $-50 \mathrm{dBm}$ (blue, green, yellow, and red, respectively), dashed lines are simulations. The black curve is obtained when spins have been saturated by an initial strong pulse. The curves have been rescaled by $\sqrt{P_{\text {in }} / P_{0}}$ for easier comparison, with $P_{0}=-90 \mathrm{dBm}$. (c) In-phase (blue) and out-of-phase (green) quadrature of the reflected field for $P_{\text {in }}=-90 \mathrm{dBm}$. (d) Spin-echo sequence. An incoming microwave pulse $\theta$ with power $-60 \mathrm{dBm}$ is followed by a delay $\tau$ and a 1- $\mu$ s-long refocusing pulse $(R)$ with power $-20 \mathrm{dBm}$, yielding an echo $e$ at time $2 \tau$. Saturation of the amplifiers (shown in red) limits the measurable amplitude to about 2 V. (e) Experimental (crosses) and simulated and rescaled (open circles) area of the echo as a function of the refocusing pulse power $P_{R}$. (f) Measured (crosses) decay of the echo maximum amplitude as a function of $\tau$. Dashed and solid lines are an exponential fit yielding a characteristic time $T_{2}=8.4 \mu \mathrm{s}$ and a biexponential fit $f(\tau)$ yielding $T_{2 A}=4.7 \mu \mathrm{s}$ and $T_{2 B}=14.3 \mu \mathrm{s}$, respectively. 
generated by the planar resonator [see Fig. 1(b)], which causes a spread of Rabi frequency within the ensemble; in particular, this prevents the application of precise $\pi$ pulses to all the spins [34], which is an issue for Hahn-echo sequences.

In order to understand in detail the spin dynamics, we compare the experimental data to the result of numerical simulations. These simulations consist of a number of mean-value equations along the lines of Ref. [14] and explained in further detail in the Supplemental Material [28]. In particular, the inhomogeneity in both spin frequency and coupling strength is taken into account by dividing the ensemble into a sufficiently large set of homogeneous subensembles and integrating the equations of motion for the resonator field and the spin components of all of the subensembles. The distribution of spin frequencies follows from the spin susceptibility shown in Fig. 5(a), and the distribution of coupling strengths depends on the resonator-field vacuum fluctuations, whose spatial distribution is calculated using the COMSOL simulation package and exemplified in the inset of Fig. 1(b). The actual distributions used are shown in Fig. S5 of the Supplemental Material [28].

The simulations employed assume an ensemble of spin$1 / 2$ particles, which is an approximation in the case of $\mathrm{NV}$ centers having a spin of 1 . However, in the linear, nonsaturated regime this description is exact, and for the nonlinear, saturated regime we expect the approximation to be justified since the applied $\pi$ pulse has a narrow frequency bandwidth and is tuned predominantly to the $|0\rangle \rightarrow|+\rangle$ transition of the NV centers. In Figs. 4(b) and 4(c), the measured and calculated reflected field are compared and show a convincing agreement, without any adjustable parameter. This confirms the validity of the calculations, both in the linear and nonlinear regime, and proves, in particular, that the frequency distribution used is correct.

\section{SPIN ECHO AT HIGH POWER}

Despite the impossibility of applying well-defined $\pi$ pulses to the spins, we implement a spin-echo sequence with an initial microwave pulse creating a transverse magnetization, followed after $\tau$ by a refocusing pulse. Its power $P_{R}=-20 \mathrm{dBm}$ is chosen such that spin saturation is reached within the pulse duration, as required for spin echo. The reflected signal amplitude is shown in Fig. 4(d), with the expected spin echo observed at $2 \tau$. We study the amplitude of this echo as a function of $P_{R}$, and compare this curve to the result of the simulations. The agreement is quantitative, as shown in Fig. 4(e); in particular, the power at which the echo amplitude saturates is well predicted by the simulations. This shows further evidence of the validity of calculated coupling strengths and of the spin-1/2 approximation.

The dependence of the echo amplitude on $\tau$ is fitted by a biexponential function $f(\tau)=A \exp \left(-2 \tau / T_{2 A}\right)+$ $B \exp \left(-2 \tau / T_{2 B}\right)$, with two different coherence times
$T_{2 A}=4.8 \mu \mathrm{s}$ and $T_{2 B}=14.3 \mu \mathrm{s}$, and $A=0.78$ and $B=$ 0.22 [see Fig. 4(f)]. Such a dependence is expected for an ensemble of NV centers in zero magnetic field. Indeed, the coherence time of NV centers is limited by dipolar interactions with the surrounding spin bath, either paramagnetic impurities ( $\mathrm{P} 1$ centers) or ${ }^{13} \mathrm{C}$ nuclear spins. This spin bath can be approximated as generating a fluctuating magnetic field that blurs the phase of the NV center. In zero magnetic field, an interesting situation occurs: the nuclear spin state $m_{I}=0$ becomes immune to first order to magnetic fluctuations [31] because of the strain-induced coupling between states $m_{S}= \pm 1$, which gives rise to an avoided level crossing, and thus to a transition frequency independent of magnetic field to first order [see Fig. 1(d)]. This was shown in previous work to make the freeinduction decay time $T_{2}^{*} 1$ order of magnitude longer in zero magnetic field [31], and should equally lead to a longer Hahn-echo time $T_{2}$. However, this is not true for states with $m_{I}= \pm 1$, which should, therefore, have a shorter decoherence time $T_{2}$ in zero magnetic field. More details will be given in future work.

\section{MULTIMODE 2PE PROTOCOL AND DISCUSSION}

Finally, we implement the multimode 2PE protocol with weak microwave pulses. Six consecutive microwave pulses with a varying phase and identical amplitude corresponding to $\approx 10^{4}$ photons in the resonator are first absorbed by the spin ensemble, and a strong refocusing pulse is then applied $10 \mu$ s later [see Fig. 5(a)]. The sequence is averaged $10^{4}$ times at a repetition rate of $1 \mathrm{~Hz}$, made possible by the active reset of the spins. As shown in Fig. 5(b), the six pulses are recovered after the refocusing pulse up to $35 \mu \mathrm{s}$ after their storage, with an amplitude reduced by $\sim 10^{2}$ compared to the incoming pulse, corresponding to $\sim 1$ photon in the resonator. As expected, the pulses are reemitted in reverse order [see Fig. 5(c)]. Note that the strong refocusing pulse $\left(\sim 10^{9}\right.$ photons in the cavity) does not prevent detection of fields at the single-photon level few microseconds later. We were able to detect a measurable spin-echo signal for pulses containing up to 100 times lower energy, thus populating the resonator with $\bar{n} \approx 100$ photons on average [see Fig. 5(d)].

An important figure of merit is the field retrieval efficiency $E$, defined as discussed in the Introduction as the ratio between the energy recovered during the echo and the energy of the incoming pulse. In the data shown in Fig. 5(b), $E$ is seen to decrease with $\tau$ due to spin decoherence, following approximately the relation $E_{e}=0.03|f(\tau)|^{2}$, which yields $E=2.4 \times 10^{-4}$ for $2 \tau=17 \mu$ s. Coming back to the figures of merit defined in the Introduction, our measurements reach $n_{\mathrm{mw}} \approx 3$, $n_{\mathrm{sp}} \approx 0.1, \bar{n} \approx 100$, and $E \approx 2 \times 10^{-4}$, many orders of magnitude closer to the quantum regime than previous state-of-the-art experiments [25]. 
Reaching the quantum regime, however, requires a recovery efficiency $E$ close to 1, and, therefore, calls for a quantitative understanding of our measurement imperfections. For that purpose we perform simulations of the multimode 2PE protocol. As seen in Fig. 5(b), the measurements are well reproduced, although a 7 times higher efficiency $E_{t}=0.21|f(\tau)|^{2}$ is predicted. We attribute the discrepancy between $E_{e}$ and $E_{t}$ to the imperfect modeling of decoherence. Indeed, our simulations treat spin decoherence in the Markov approximation. This is not an adequate treatment since it is well known that the spin bath environment displays strong memory effects. In particular, this Markov approximation is expected to improperly describe the dynamics of a spin under the action of a microwave drive, as happens during the refocusing pulse. This non-Markovian bath causes the Rabi oscillation of a single spin to decay faster than the spin-echo damping time $T_{2}$, as was observed in Ref. [35], for instance. This effect is not included in our simulations and might explain the remaining discrepancy between theory and measurements. Overall, we infer from the simulations that $E_{t}$ would reach 0.2 for a sample with infinite $T_{2}$; this number quantifies the reduced efficiency caused by refocusing pulse imperfections and finite spin absorption. In the measured efficiency $E_{e} \approx 2 \times 10^{-4}$, finite spin coherence causes a further $10^{-3}$ reduction, thus appearing as the main limitation of the field retrieval efficiency in the present experiment.

According to the previous analysis, a 1 order of magnitude increase of the coherence time would be sufficient to reach an echo efficiency $\approx 0.1$, which would enable first experiments in the quantum regime. This can be achieved [9] with samples having a reduced concentration of nitrogen paramagnetic impurities as well as isotopic enrichment of ${ }^{12} \mathrm{C}$. Further improvements of the echo efficiency will be reached thanks to better refocusing pulses using rapid adiabatic passage [14,36], or by tailoring the spin spatial distribution [37]. These combined advances should make it possible to reach the figures of merit required to implement a complete quantum memory protocol $[13,14]$ at the single-photon level and to experimentally explore its fidelity. Optical pumping in a hybrid circuit, as demonstrated here, is also a first step towards the polarization of the nitrogen nuclear spins [38], and in the longer term towards a nuclear-spin-based quantum memory.

In conclusion, we implement the multimode storage and retrieval of microwave fields in an ensemble of NV centers in diamond at millikelvin temperatures, with active reset by optical pumping and refocusing by a strong microwave pulse. These results demonstrate that complex dynamical control of spin ensembles is compatible with hybrid
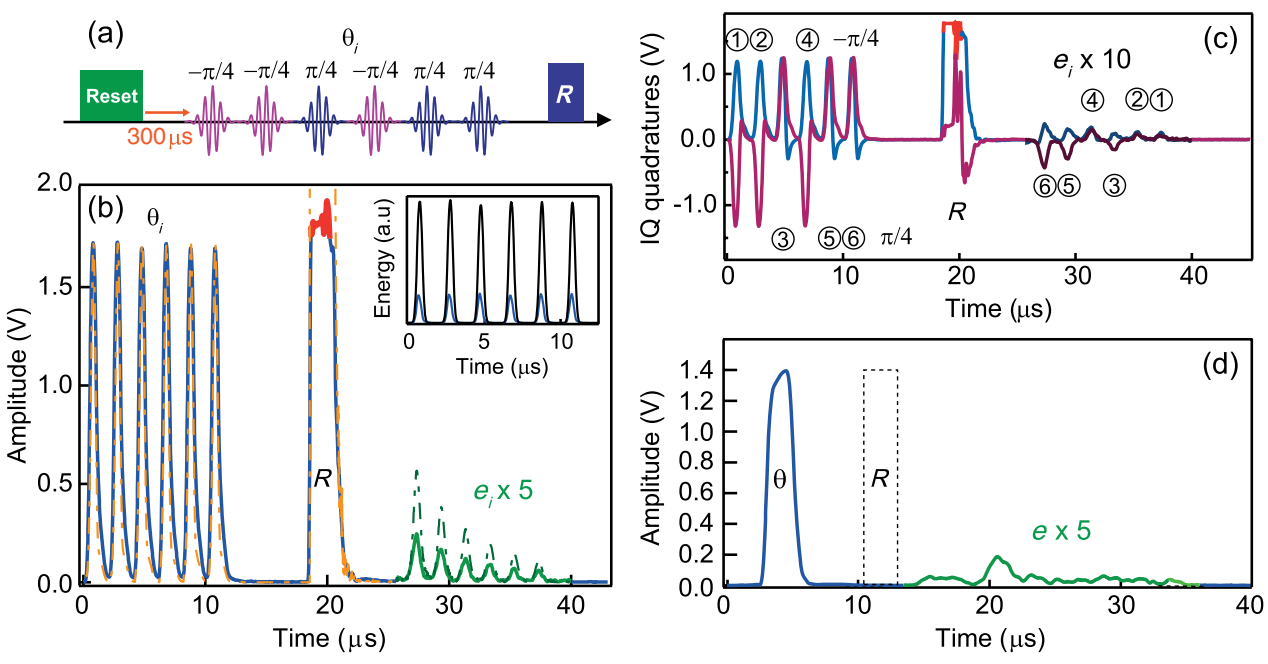

FIG. 5. Test of the 2PE protocol for multimode storage of few-photon pulses. (a) Experimental sequence including a spin reset pulse, a train of six microwave pulses $\theta_{i}(i=1, \ldots, 6)$ with an identical amplitude (corresponding to $\sim 10^{4}$ photons in the resonator) and different phases $\varphi_{1}=\varphi_{2}=\varphi_{4}=-\pi / 4$ and $\varphi_{3}=\varphi_{5}=\varphi_{6}=\pi / 4$, and a $50 \mathrm{~dB}$ stronger refocusing pulse $R$ with phase $\varphi_{r}=0.1 \mathrm{rad}$. (b) Amplitude of the measured (solid line) and calculated (dash-dotted line) output signal showing the reflected pulses $\theta_{i}$ (after partial absorption by the spins) and $R$ (its amplitude being trimmed by amplifier saturation, shown in red), as well as the six reemitted echoes $e_{i}$ (magnified by a factor of 5). Inset: Comparison between the energies of the reflected $\theta_{i}$ pulses with the spins saturated (black line) or reset in their ground state (blue line) shows that about $75 \%$ of the incident power is absorbed by the spins. (c) In-phase (blue solid line) and Out-of-Phase (purple solid line) quadratures I and Q of the output signal, showing that the $e_{i}$ pulses (magnified by 10) are recovered with phase $-\left(\varphi_{i}-\varphi_{r}\right)$, as expected. (d) Spin echo $e$ of $\sim 0.02$ photons in the resonator for a lowpower incoming $\theta$ pulse populating the resonator with only $\sim 100$ photons. The refocusing pulse (dashed line) was suppressed in the room-temperature detection chain by a microwave switch to avoid saturating the follow-up amplifiers. 
quantum circuits, thus enabling the long-term storage of quantum information in electronic or nuclear spin ensemble quantum memory.

\section{ACKNOWLEDGMENTS}

We acknowledge technical support from P. Sénat, D. Duet, J.-C. Tack, P. Pari, and P. Forget, as well as useful discussions within the Quantronics group and with A. Dréau, J.-F. Roch, T. Chanelière, and J. Morton. We acknowledge support from the French National Research Agency (ANR) with the QINVC project from CHISTERA program, the European project SCALEQIT, and the C'Nano IdF project QUANTROCRYO. Y. K. is supported by the Japanese Society for the Promotion of Science (JSPS). B. J. and K. M. acknowledge support from the Villum Foundation.

[1] B. Julsgaard, J. Sherson, J. I. Cirac, J. Fiurasek, and E. S. Polzik, Experimental Demonstration of Quantum Memory for Light, Nature (London) 432, 482 (2004).

[2] A. I. Lvovsky, B. C. Sanders, and W. Tittel, Optical Quantum Memory, Nat. Photonics 3, 706 (2009).

[3] C. Clausen, I. Usmani, F. Bussires, N. Sangouard, M. Afzelius, H. de Riedmatten, and N. Gisin, Quantum Storage of Photonic Entanglement in a Crystal, Nature (London) 469, 508 (2011).

[4] V. Damon, M. Bonarota, A. Louchet-Chauvet, T. Chanelière, and J.-L. Le Gouët, Revival of Silenced Echo and Quantum Memory for Light, New J. Phys. 13, 093031 (2011).

[5] D. L. McAuslan, P. M. Ledingham, W. R. Naylor, S. E. Beavan, M.P. Hedges, M.J. Sellars, and J. J. Longdell, Photon-Echo Quantum Memories in Inhomogeneously Broadened Two-Level Atoms, Phys. Rev. A 84, 022309 (2011).

[6] K. Tordrup, A. Negretti, and K. Mølmer, Holographic Quantum Computing, Phys. Rev. Lett. 101, 040501 (2008).

[7] K. Tordrup and K. Mølmer, Quantum Computing with a Single Molecular Ensemble and a Cooper-Pair Box, Phys. Rev. A 77, 020301(R) (2008).

[8] J. H. Wesenberg, A. Ardavan, G. A. D. Briggs, J. J. L. Morton, R. J. Schoelkopf, D. I. Schuster, and K. Mølmer, Quantum Computing with an Electron Spin Ensemble, Phys. Rev. Lett. 103, 070502 (2009).

[9] N. Bar-Gill, L. M. Pham, A. Jarmola, D. Budker, and R. L. Walsworth, Solid-State Electronic Spin Coherence Time Approaching One Second, Nat. Commun. 4, 1743 (2013).

[10] A. Tyryshkin, S. Tojo, J. Morton, H. Riemann, N. Abrosimov, P. Becker, H.-J. Pohl, T. Schenkel, M. Thewalt, K. Itoh, and S. Lyon, Electron Spin Coherence Exceeding Seconds in High-Purity Silicon, Nat. Mater. 11, 143 (2012).

[11] M. Steger, K. Saeedi, M. L. W. Thewalt, J. J. L. Morton, H. Riemann, N. V. Abrosimov, P. Becker, and H.-J. Pohl, Quantum Information Storage for over 180 s Using Donor Spins in a ${ }^{28} \mathrm{Si}$ "Semiconductor Vacuum", Science 336, 1280 (2012).
[12] J. T. Muhonen, J. P. Dehollain, A. Laucht, F. Hudson, T. Sekiguchi, K. Ioth, D. N. Jamieson, J. C. McCallum, A.S. Dzurak, and A. Morello, Storing Quantum Information for 30 Seconds in a Nanoelectronic Device, arXiv:1402.7140.

[13] M. Afzelius, N. Sangouard, G. Johansson, M. U. Staudt, and C. M. Wilson, Proposal for a Coherent Quantum Memory for Propagating Microwave Photons, New J. Phys. 15, 065008 (2013).

[14] B. Julsgaard, C. Grezes, P. Bertet, and K. Mølmer, Quantum Memory for Microwave Photons in an Inhomogeneously Broadened Spin Ensemble, Phys. Rev. Lett. 110, 250503 (2013).

[15] Y. Kubo, F. R. Ong, P. Bertet, D. Vion, V. Jacques, D. Zheng, A. Dreau, J. F. Roch, A. Auffeves, F. Jelezko, J. Wrachtrup, M. F. Barthe, P. Bergonzo, and D. Esteve, Strong Coupling of a Spin Ensemble to a Superconducting Resonator, Phys. Rev. Lett. 105, 140502 (2010).

[16] D. I. Schuster, A. P. Sears, E. Ginossar, L. DiCarlo, L. Frunzio, J. J. L. Morton, H. Wu, G. A. D. Briggs, B. B. Buckley, D. D. Awschalom, and R. J. Schoelkopf, High-Cooperativity Coupling of Electron-Spin Ensembles to Superconducting Cavities, Phys. Rev. Lett. 105, 140501 (2010).

[17] R. Amsüss, C. Koller, T. Nöbauer, S. Putz, S. Rotter, K. Sandner, S. Schneider, M. Schramböck, G. Steinhauser, H. Ritsch, J. Schmiedmayer, and J. Majer, Cavity QED with Magnetically Coupled Collective Spin States, Phys. Rev. Lett. 107, 060502 (2011).

[18] V. Ranjan, G. de Lange, R. Schutjens, T. Debelhoir, J. P. Groen, D. Szombati, D. J. Thoen, T. M. Klapwijk, R. Hanson, and L. DiCarlo, Probing Dynamics of an Electron-Spin Ensemble via a Superconducting Resonator, Phys. Rev. Lett. 110, 067004 (2013).

[19] S. Probst, H. Rotzinger, S. Wünsch, P. Jung, M. Jerger, M. Siegel, A. V. Ustinov, and P. A. Bushev, Anisotropic RareEarth Spin Ensemble Strongly Coupled to a Superconducting Resonator, Phys. Rev. Lett. 110, 157001 (2013).

[20] E. L. Hahn, Spin Echoes, Phys. Rev. 80, 580 (1950).

[21] B. Julsgaard and K. Mølmer, Fundamental Limitations in Spin-Ensemble Quantum Memories for Cavity Fields, Phys. Rev. A 88, 062324 (2013).

[22] Y. Kubo, C. Grezes, A. Dewes, T. Umeda, J. Isoya, H. Sumiya, N. Morishita, H. Abe, S. Onoda, T. Ohshima, V. Jacques, A. Dréau, J.-F. Roch, I. Diniz, A. Auffeves, D. Vion, D. Esteve, and P. Bertet, Hybrid Quantum Circuit with a Superconducting Qubit Coupled to a Spin Ensemble, Phys. Rev. Lett. 107, 220501 (2011).

[23] X. Zhu, S. Saito, A. Kemp, K. Kakuyanagi, S.-i. Karimoto, H. Nakano, W. J. Munro, Y. Tokura, M. S. Everitt, K. Nemoto, M. Kasu, N. Mizuochi, and K. Semba, Coherent Coupling of a Superconducting Flux Qubit to an Electron Spin Ensemble in Diamond, Nature (London) 478, 221 (2011).

[24] A. G. Anderson, R. L. Garwin, E. L. Hahn, J. W. Horton, G. L. Tucker, and R. M. Walker, Spin Echo Serial Storage Memory, J. Appl. Phys. 26, 1324 (1955).

[25] H. Wu, R. E. George, J. H. Wesenberg, K. Mølmer, D. I. Schuster, R. J. Schoelkopf, K. M. Itoh, A. Ardavan, J. J. L. Morton, and G. A. D. Briggs, Storage of Multiple Coherent 
Microwave Excitations in an Electron Spin Ensemble, Phys. Rev. Lett. 105, 140503 (2010).

[26] N. B. Manson, J. P. Harrison, and M. J. Sellars, NitrogenVacancy Center in Diamond: Model of the Electronic Structure and Associated Dynamics, Phys. Rev. B 74, 104303 (2006).

[27] Y. Kubo, I. Diniz, A. Dewes, V. Jacques, A. Dréau, J.-F. Roch, A. Auffeves, D. Vion, D. Esteve, and P. Bertet, Storage and Retrieval of a Microwave Field in a Spin Ensemble, Phys. Rev. A 85, 012333 (2012).

[28] See Supplemental Material at http://link.aps.org/ supplemental/10.1103/PhysRevX.4.021049 for details on the experimental setup and on the theoretical model.

[29] P. Neumann, R. Kolesov, V. Jacques, J. Beck, J. Tisler, A. Batalov, L. Rogers, N. B. Manson, G. Balasubramanian, F. Jelezko, and J. Wrachtrup, Excited-State Spectroscopy of Single NV Defects in Diamond Using Optically Detected Magnetic Resonance, New J. Phys. 11, 013017 (2009).

[30] S. Felton, A. M. Edmonds, M. E. Newton, P. M. Martineau, D. Fisher, D. J. Twitchen, and J. M. Baker, Hyperfine Interaction in the Ground State of the Negatively Charged Nitrogen Vacancy Center in Diamond, Phys. Rev. B 79, 075203 (2009).

[31] F. Dolde, H. Fedder, M. W. Doherty, T. Nbauer, F. Rempp, G. Balasubramanian, T. Wolf, F. Reinhard, L. C. L. Hollenberg, F. Jelezko, and J. Wrachtrup, Electric-Field Sensing Using Single Diamond Spins, Nat. Phys. 7, 459 (2011).

[32] B. Julsgaard and K. Mølmer, Dynamical Evolution of an Inverted Spin Ensemble in a Cavity: Inhomogeneous
Broadening as a Stabilizing Mechanism, Phys. Rev. A 86, 063810 (2012).

[33] L. Robledo, L. Childress, H. Bernien, B. Hensen, P. Alkemade, and R. Hanson, High-fidelity Projective ReadOut of a Solid-State Spin Quantum Register, Nature (London) 477, 574 (2011).

[34] H. Malissa, D. I. Schuster, A. M. Tyryshkin, A. A. Houck, and S. A. Lyon, Superconducting Coplanar Waveguide Resonators for Low Temperature Pulsed Electron Spin Resonance Spectroscopy, Rev. Sci. Instrum. 84, 025116 (2013).

[35] R. Hanson, O. Gywat, and D. D. Awschalom, RoomTemperature Manipulation and Decoherence of a Single Spin in Diamond, Phys. Rev. B 74, 161203 (2006).

[36] A. J. Sigillito, H. Malissa, A. M. Tyryshkin, H. Riemann, N. V. Abrosimov, P. Becker, H.-J. Pohl, M. L. W. Thewalt, K. M. Itoh, J. J. L. Morton, A. A. Houck, D. I. Schuster, and S. A. Lyon, Fast, Low-Power Manipulation of Spin Ensembles in Superconducting Resonators, arXiv:1403.0018.

[37] O. Benningshof, H. Mohebbi, I. Taminiau, G. Miao, and D. Cory, Superconducting Microstrip Resonator for Pulsed ESR of Thin Films, J. Magn. Reson. 230, 84 (2013).

[38] V. Jacques, P. Neumann, J. Beck, M. Markham, D. Twitchen, J. Meijer, F. Kaiser, G. Balasubramanian, F. Jelezko, and J. Wrachtrup, Dynamic Polarization of Single Nuclear Spins by Optical Pumping of Nitrogen-Vacancy Color Centers in Diamond at Room Temperature, Phys. Rev. Lett. 102, 057403 (2009). 